\title{
Design of Service Robot for Wearing and Taking off Footwear
}

\author{
Yunsheng Li ${ }^{1}$, Anjian Xiao ${ }^{1}$, Quanquan Feng ${ }^{1}$, Tian Zou ${ }^{1}$ and Chunlai Tian ${ }^{*}$ \\ ${ }^{1}$ Pingxiang City Key Laboratory of High Gravity Process Intensification, School of Mechanical and Electronic Engineering, Pingxiang \\ University, Pingxiang City, Jiangxi Province, 337000, China
}

\begin{abstract}
In order to realize the automation and integration of wearing and taking off shoes and socks, a service robot for wearing and taking off footwear was designed to help persons living with waist or leg problems in this paper. Through the analysis of the process of wearing and taking off shoes and socks, the robot could carry out this work automatic. The robot mainly consists of intelligent identification system, device for wearing and removing shoes or socks, mobile mechanical arm and power drive with control system. In addition, an auxiliary foot lifting device is designed to cooperate with the service robot for wearing and taking off footwear. The virtual motion simulation of the machine is carried out, and the feasibility of the machine function and the rationality of the structure design are analyzed here.
\end{abstract}

\section{Introduction}

Wearing and taking off shoes and socks is a common thing for people, but it is a painful thing for the elderly, patients, the disabled and other people with waist or leg illness. The special home service robot is one type of the assistive robots to help them in normal living [1-4]. There are a few designs on automatic service robots for dressing and wearing shoes or socks at present [5-9]. They are mostly helpful for dressing and wearing shoes or socks. An automatic service robot, which realizes the integration of putting on and taking off shoes and socks, is designed in this paper. The robot has guiding value for the popularization and market-oriented development of the old-age service for our residents, and provides great convenience for users.

\section{Overall design and structure}

The service robot realizes four processes of automatic shoe-wearing, shoe-taking, sock-wearing and sock-taking. Firstly, user needs to place shoes and apparatus fixed socks in the room. When the robot receives the action signal properly, the movable arm grabs shoes and apparatus fixed socks separately and puts them in the robot's position. The shoes are placed on the shoe tray of the device for wearing and removing shoes. The apparatus for fixing socks is placed in the device for wearing and removing socks. Then the robot will travel to the user's legs area. After it is positioned accurately by the intelligent recognition system, it will start to perform sock-wearing and shoe-wearing actions. The overall structure of the robot is shown in Fig. 1 and the system connection of the whole machine labelled is shown in Fig. 2. It is mainly divided into seven modules: mobile mechanical arm, intelligent recognition system, device for wearing and removing shoes, device for wearing and removing socks, top cover device, walking system and power and control system. From the perspective of actions, it is divided into the prime mover part, the transmission part, the execution part, the control and the auxiliary system.

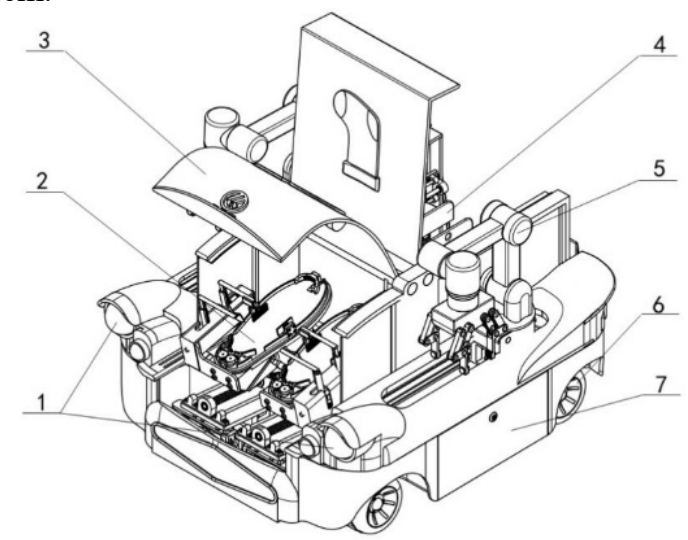

Figure 1. The overall structure of robot.

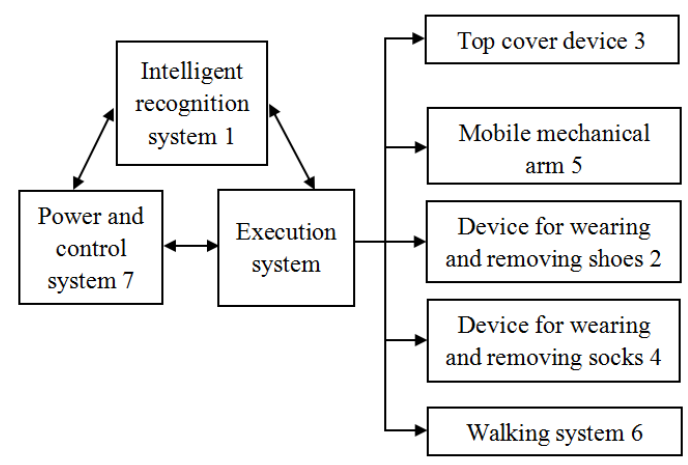

Figure 2. System association of robot.

*tianchunlai@bit.edu.cn 


\section{Part design}

\subsection{Device for wearing and removing shoes}

The device for wearing and removing shoes need to simulate the two processes of wearing and removing shoes in real life. The overall structure is shown in Fig. 3. It is divided into five parts: shoe tray clamping mechanism, shoe mouth expanding mechanism, shoe lifting mechanism, shoe parallel moving mechanism and shoe pushing mechanism. The shoe tray clamping mechanism is used to solve the problem of shoe placement and fixing. The shoe mouth expanding mechanism is used to prevent the collapse of the shoe tongue and the pressing of the heel in the process of wearing shoes, so as to realize the expansion of the shoe mouth. The shoe lifting mechanism is used to adjust the angle and height of the shoe tray, and it includes the scissors lifting mechanism and the screw pusher mechanism. The shoe parallel moving mechanism is used to realize the front and back positioning and the moving process of wearing and taking off shoes. The shoe pushing mechanism is used for lifting the back part of the shoe. The shoe expanding device for shoe mouth is shown in Fig. 4 for details display.

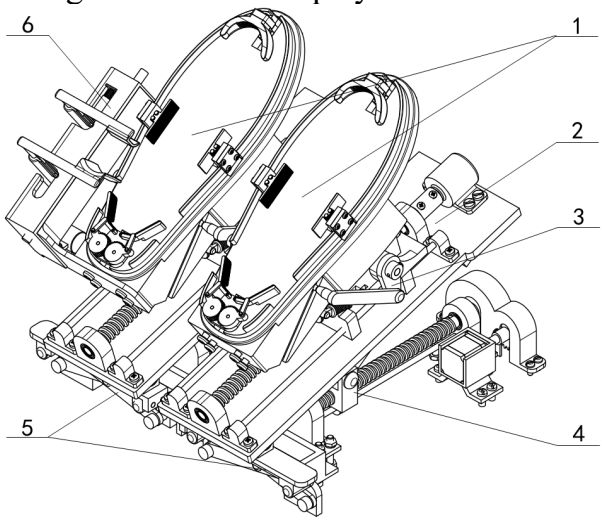

1. Shoe tray clamping mechanism 2 . Shoe parallel moving mechanism

3. Shoe pushing mechanism 4. Screw pusher 5. Lifting mechanism

6. Shoe mouth expanding

Figure 3. Wearing and removing shoes device.

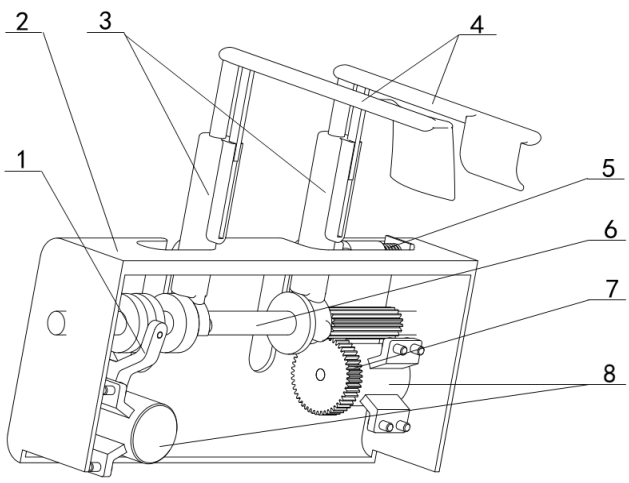

1. Swing rod 2. Shell 3. Cylinder 4. Shoe brace 5. Reset spring

6. Gear shaft 7. Gear 8. Motor

Figure 4. Shoe mouth expanding device.
As shown in Fig. 4, the device is used to enlarge the opening of the shoe before wearing the shoe, which makes it easier for the foot to insert into the shoe. The cylinder is used to lift and drop the shoe brace, which can solve the height problem of different shoes. One motor drives the gear shaft to rotate through the gear, so that the two shoe props fixed on the gear shaft can turn over. The other motor drives the gear shaft to move in a straight line through the swing rod, and the front-shoe prop can follow the gear shaft. In addition, the back-shoe prop rotates due to the action of the shell, and finally realizes the opening of the shoe mouth. After wearing shoes, the shoe braces are reset under the action of reset spring and are turned over by motor drive.

\subsection{Device for wearing and removing socks}

The device for wearing and removing socks is shown in Fig. 5. It uses sensors to realize the positioning of apparatus for fixing socks. The sock-wearing process is realized by the combination of the screw drive and apparatus for fixing socks, and the sock-removing process is realized by the combination of the screw drive and sock remover. Many pairs of flexible socks are fixed in advance and reversely on apparatus for fixing socks and placed in the designated position. The screw drive mechanism can realize the plane movement of the apparatus for fixing socks and the sock remover. The mechanism composed of worm and worm wheel controls the rotation of apparatus for fixing socks and sock remover.

The sock remover is driven by the motor. The output shaft of motor is connected with two arc connecting rods. The arc connecting rods are connected with two symmetrical sock removing rods. The sock removing rods are connected to the guide rail by rolling through roller. The output shaft of the motor rotates to pull the two sock removing rods to move in opposite direction or back direction. A distance sensor is arranged on the sock removed rod to precisely control the configured start and stop of the motor.

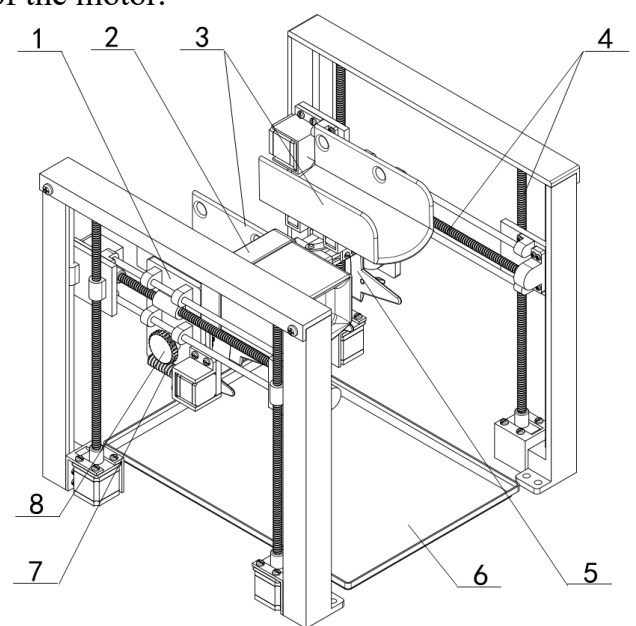

1. Mobile board 2. Sock 3. Apparatus for fixing socks 4. Screw drive

5. Sock remover 6. Sock plate 7. Worm 8 . Worm wheel

Figure 5. Wearing and removing socks device. 


\subsection{Auxiliary foot lifting device}

Auxiliary foot lifting device is designed for people who are unable to lift their feet for a long time. It is an auxiliary tool for wearing and taking off footwear. It is shown in Fig. 6. The device adopts the principle of pneumatic chair, and adjusts the height of adjusting rod, which solves the problem of different bed heights. At the top of the support rod, there is a double head motor, which is used to drive the soft pad to rotate, so as to lift the lower leg of a person. The two slots of the soft pad for legging will be directly opposite to the two shoe trays of the robot. Stepping motor can drive rack to adjust the position of footrest, which solves the problem of different leg length for different people. The surface design improves the comfort and the footrest can be changed by users.

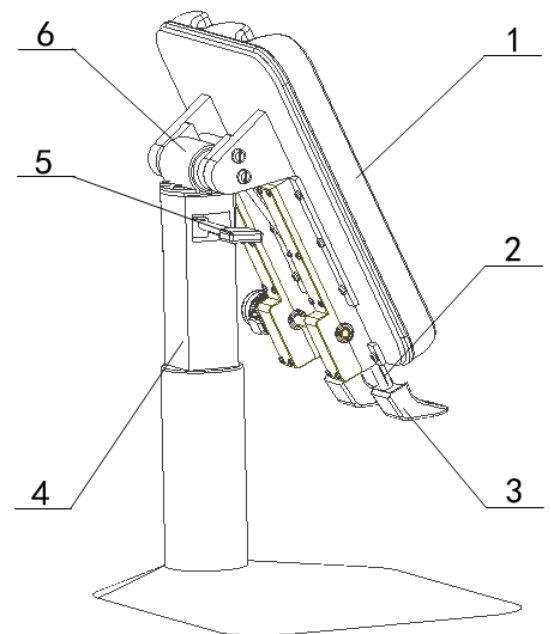

1. Soft pad 2. Rack 3. Footrest 4. Support rod 5. Adjusting rod 6. Double head motor

Figure 6. Auxiliary foot lifting device.

\subsection{Mobile mechanical arm}

The mobile mechanical arm is located on both sides of the robot, which is used to grasp and place shoes and apparatus for fixing socks. The overall movement of the mechanical arm is precisely controlled by gear, rack and hub. It can realize multi-degree-of-freedom motion by itself. Manipulator gripper adopts worm gear and worm mechanism and parallel four-link mechanism, and the two motors are independently controlled, which solves the problem of different front and rear width of shoes. The mechanical claw uses rubber material to contact with shoes, soft and antiskid.

\subsection{Walking and control system}

The four wheels in the walking system are equipped with two motors. One motor drives the wheel to rotate inside the hub, the other motor drives the wheel steering above the robot chassis. The steering system adopts bevel gear drive. Through the control system, the four wheels of the robot cooperate with each other, and finally realize the omni-directional movement of the robot, which can rotate in situ.
The robot uses the combination of PLC control system and various sensors. The sensor mainly uses vision sensor, distance sensor and pressure sensor. PLC is a special software to write control programs to achieve control, the application of PLC instructions to pneumatic devices, stepper motor drivers and DC for algorithm control, through each link of the corresponding sensor feedback information for algorithm adjustment to achieve accurate and reliable control effect.

\section{Conclusion}

The household service robot for wearing and taking off footwear is designed here for the automation of wearing and taking off shoes and socks for people. It has a good value for the popularization and market development of residents' elderly care services. It can provide great living convenience for pregnant women, patients, and the disabled. The design of this robot is based on a reasonable mechanical structure and cooperates with the control principle. It has novel structure and adopts modular design. Its prototype design with test will be carried out in the future research.

\section{Acknowledgment}

Thanks to Prof. Jun Zhang, Prof. Ping He and Dr. Honggang Zhang in Pingxiang City Key Laboratory of High Gravity Process Intensification, School of Mechanical and Electronic Engineering, Pingxiang University for the manufacturing process advice in the prototype design.

National Key R\&D Program of China (No. 2016YFC0209305), Science and Technology Planning Projects of Jiangxi Province (No. 20181BBG78075) and Scientific Research Innovation Team Support Project of Pingxiang University.

\section{References}

1. Robinson, H., Macdonald, B., Broadbent, E., "The role of healthcare robots for older people at home: a review," Int J Soc Robot, vol 6, pp. 575-591, 2014.

2. Jevtic, A., Valle, A. F., Alenya, G., Chance, G., Caleb-Solly, P. Dogramadzi, S., "Personalized robot assistant for support in dressing," IEEE T Cogn Dev Sys, vol 99, pp. 1-12, 2018.

3. Lee, M., Murata, K., Ameyama, K., Yamazoe, H., Lee, J.-H., "Development and quantitative assessment of an elbow joint robot for elderly care training," Intel Serv Robotics, vol 12, pp. 277-287, 2019.

4. Bedaf, S., Witte, L. D., "Robots for Elderly Care: Their Level of Social Interactions and the Targeted End User," Stu Health T \& Inf, vol 242, pp. 472-478, 2017.

5. Do, H. M., Pham, M., Sheng, W., Dan, Y., Liu, M., "RiSH: A robot-integrated smart home for elderly care," Robot Auton Syst, vol 101, pp. 74-92, 2017. 
6. Kapusta, A., Erickson, Z., Clever, H.M., "Personalized collaborative plans for robot-assisted dressing via optimization and simulation," Auton Robot, vol 43, pp. 2183-2207, 2019.

7. Bedaf, S., Marti, P., Amirabdollahian, F., "A multiperspective evaluation of a service robot for seniors: the voice of different stakeholders," Disabil Rehabil Assist Technol, vol 13, pp. 592-599, 2017.
8. Chen, M., Liu, C., Du, G., "A human-robot interface for mobile manipulator," Intel Serv Robotics, vol 11, pp. 1-10, 2018.

9. Kalani, H., Akbarzadeh, A., Nabavi, S. N., Moghimi, S., "Dynamic modeling and CPG-based trajectory generation for a masticatory rehab robot," Intel Serv Robotics, vol 11, pp. 1-19, 2018. 